\title{
Consensus Guides on Stroke Thrombolysis for Anticoagulated Patients from Japan: Application to Other Populations
}

\author{
Kazunori Toyoda, ${ }^{\text {a }}$ Hiroshi Yamagami, ${ }^{\mathrm{b}}$ Masatoshi Koga ${ }^{\mathrm{a}}$ \\ ${ }^{a}$ Department of Cerebrovascular Medicine, National Cerebral and Cardiovascular Center, Suita, Japan \\ ${ }^{b}$ Division of Stroke Care Unit, National Cerebral and Cardiovascular Center, Suita, Japan
}

Development of direct oral anticoagulants and their antidotes has led to the need to reconsider the eligibility of acute stroke patients who have been taking oral anticoagulants for intravenous thrombolysis. Officially authorized Japanese guidelines on this issue were revised twice at the time of approval for clinical use of direct oral anticoagulants and idarucizumab, a specific reversal agent for dabigatran. A unique recommendation in the latest Japanese clinical guides was that thrombolysis can be recommended if the time of the last dose of direct oral anticoagulants exceeds 4 hours and if commonly available anticoagulation markers are normal or subnormal, i.e., international normalized ratio of prothrombin time $<1.7$ and activated partial thromboplastin time $<1.5$ times the baseline value ( $\leq 40$ seconds only as a guide). These criteria are partly supported by the findings of domestic multicenter and single-center surveys that symptomatic or asymptomatic intracranial hemorrhage following thrombolysis was rare under the conditions of the criteria. Even for dabigatran users, stroke thrombolysis can be considered without pretreatment by idarucizumab if patients meet the above criteria. If not, direct mechanical thrombectomy can be considered without pretreatment by idarucizumab or thrombolysis, and use of idarucizumab, followed immediately by thrombolysis, can be considered only when thrombectomy cannot be quickly performed. These clinical guides are practical and to some extent economical, but they have some limitations, including lack of corroborating information from sufficient numbers of relevant cases. The guides will be further modified based on the results of future research.

Keywords Guide; Stroke; Anticoagulants; Atrial fibrillation; Idarucizumab; Thrombolyic therapy
Correspondence: Kazunori Toyoda Department of Cerebrovascular Medicine, National Cerebral and Cardiovascular Center, 5-7-1 Fujishirodai, Suita 565-8565, Japan

Tel: +81-6-6833-5012

Fax: $+81-6-6835-5267$

E-mail: toyoda@ncvc.go.jp

Received: June 17, 2018 Revised: July 19, 2018 Accepted: August 13, 2018

\section{Introduction}

There are apparent differences in responsiveness to intravenous thrombolysis (IVT) and anticoagulation between Asians, or more strictly speaking Japanese, and other populations. ${ }^{1,2}$ For example, the officially approved dosage of intravenous alteplase in IVT for stroke is $0.6 \mathrm{mg} / \mathrm{kg}$ in Japan versus $0.9 \mathrm{mg} / \mathrm{kg}$ in other countries according to the results of a domestic trial. ${ }^{3,4}$ An international normalized ratio (INR) of prothrombin time of 1.6 to 2.6 is officially recommended for stroke patients aged $\geq 70$ years with non-valvular atrial fibrillation (NVAF) in Japan; 4,5 this target range, which is lower than the global recommendation, was also found to be safe and effective as primary prevention for thromboembolic events. ${ }^{6}$ Furthermore, the Japa- 
nese official dosage of rivaroxaban for NVAF patients, ${ }^{7}$ as well as that of prasugrel for patients with coronary artery disease, ${ }^{8}$ is also lower than the official Western dosage. The differences in dosing regimens are partly due to a higher risk of intracranial hemorrhage $(\mathrm{ICH})$ as a complication and partly due to different metabolic activity for anticoagulants. ${ }^{1,29-11}$ Thus, distinctive strategies for IVT specific to Japanese patients are necessary, in particular guidelines for IVT during anticoagulant medication.

In this review, the rationale and the follow-up confirmation of the Japanese guidelines and the recent guides ${ }^{12-14}$ on stroke IVT for anticoagulated patients are discussed.

\section{Previous strategies for anticoagulated patients in the initial (2005) and second (2012) editions of the Japanese guidelines on stroke thrombolysis}

Intravenous alteplase was officially approved for clinical use in hyperacute ischemic stroke in 2005 on the basis of the results of the Japan Alteplase Clinical Trial (J-ACT) with a dosage of $0.6 \mathrm{mg} / \mathrm{kg}$ in Japan, ${ }^{3} 9$ years after approval in the United States. The Japan Stroke Society published the "Guidelines for intravenous application of rt-PA (alteplase), October 2005"12 and organized training sessions for proper use of recombinant tissue-type plasminogen activator (rt-PA) using these guidelines as the standard all over Japan in an effort to promote the safe implementation of this therapy nationwide. Concern about a higher risk of post-IVT ICH partly led to both a long delay in regulatory approval and a distinctive low-dosage of alteplase in clinical use. Alteplase at a dose of $0.6 \mathrm{mg} / \mathrm{kg}$ has an identical intensity as duteplase at a dose of 20 mega unit (MU)/kg for patients weighing $60 \mathrm{~kg}$, an optimal regimen in a domestic dose-escalation trial for hyperacute stroke. ${ }^{15}$ The optimal dosage of intravenous alteplase for acute coronary syndrome in studies from Japan was around three-fifths of that from Western countries. ${ }^{16}$ The lower-dosage IVT was proven to be feasible for Japanese patients in post-marketing surveillance and registry studies ${ }^{17-19}$ and for non-Japanese populations in the recent ENhanced Control of Hypertension and Thrombolysis strokE stuDy (ENCHANTED) trial. ${ }^{20}$ In the initial edition of the Japanese special guidelines on IVT, IVT was contraindicated for stroke patients taking warfarin with INR $>1.7$ and those receiving heparin with activated partial thromboplastin time (aPTT) $>1.5$ times the baseline value. These cut-off values of anticoagulant markers followed guidelines in other countries, since patients with higher values were excluded from the J-ACT, and their safety remained unknown. ${ }^{12}$ Findings from the Get With The Guidelines-Stroke Registry study supported the validity of the cut-off INR value of 1.7.21

The second edition of the Japanese guidelines on IVT was published in 2012 concurrently with extended insurance coverage of the therapeutic time window from 3 to 4.5 hours from symptom onset. ${ }^{13}$ At the time, reports on IVT for direct oral anticoagulant (DOAC) users had been few. Expert opinions were negative towards IVT for DOAC users within 48 hours after the last dosing or unless any relevant anticoagulant activity was ruled out by thrombin time, ecarin clotting time, or the Hemoclot test if taking dabigatran and the antiXa activity test if taking factor Xa inhibitors. ${ }^{22}$ These anticoagulation tests are time-consuming or not available in regular clinical use and inappropriate for recommending as routine practice in the guidelines. Another review mentioned that IVT could be considered, for example, if 12 hours had passed from the last dosing of dabigatran, aPTT is normal, and the results of other specific anticoagulation tests are only slightly elevated, and if 6 hours had passed from the last dosing of rivaroxaban or apixaban with normal levels of aPTT and prothrombin time. ${ }^{23}$ Since alteplase had already been limited to a lower dosage in Japan than the global recommendation, the committee members of the second edition did not want to add excessively strict limitations to IVT for anticoagulated patients. The members tried to determine eligibility for IVT based on clinically-used anticoagulation tests and time from the last dosing, as in the above review. Thus, the cut-off levels of anticoagulation parameters were taken from the initial edition, so that patients should be considered ineligible for IVT if they have an INR of $>1.7$ or an aPTT of $>1.5$ times the baseline value, even when patients taking DOACs. With respect to the cut-off of the dosing-to-needle time, opinions varied among the members from no limits to 12 hours. The members finally agreed not to limit the dosing-toneedle time in the headline recommendation, but they noted in the main text that special care should be taken in determining whether the potential benefits of IVT outweigh the potential risks in patients on regular DOAC medication until approximately half a day after the time of the last dose, because DOACs have a half-life of around 12 hours, and the eligibility criteria for IVT in DOAC users will be modified based on future research outcomes. In addition, another headline recommendation noted that, for any patients on antithrombotic therapy, especially on oral anticoagulant therapy, the decision-making for IVT should be determined with care.

A full description of the previous recommendations in the second edition is shown in Table 1. 
Table 1. Previous recommendations on thrombolysis for anticoagulated patients from the Japanese Guidelines for intravenous application of recombinant tissue-type plasminogen activator, second edition, October 2012

\begin{abstract}
Contraindications
- International normalized ratio (INR) of prothrombin time $>1.7$

- Prolonged activated partial thromboplastin time (aPTT) (>1.5 times the baseline value [approximately $>40$ seconds only as a guide])

Although the first edition (2005) contained hematological criteria applicable only to patients receiving warfarin or heparin, the second edition included modified criteria for a wide range of patients prescribed any anticoagulants in light of the recent official approvals of direct oral anticoagulants (DOACs). No appropriate markers are now available to measure the intensity of direct factor Xa inhibitors (rivaroxaban, apixaban, and edoxaban) or a direct thrombin inhibitor (dabigatran). Either of INR or aPTT is not an essential marker predictive of a hemorrhagic tendency after intravenous administration of alteplase combined with DOACs, although the former somewhat reflects the intensity of $\mathrm{Xa}$ inhibitors and the latter the intensity of dabigatran. At this time, patients should be considered ineligible for IV alteplase if at least they have an INR, which is a marker of the intensity of conventional anticoagulants, of $>1.7$ or an aPTT of $>1.5$ times the baseline value (approximately $>40$ seconds only as a guide, though absolute values vary among reagents).

Careful administration* (eligibility should be determined with care)

- On thrombolytic or antithrombotic therapy (especially oral anticoagulant therapy)

Since it has not been long since DOACs in particular were started to be used in clinical practice, and the efficacy and safety of intravenous alteplase in patients taking DOACs have not been established, patient eligibility for treatment should be determined with caution. As described above, it is necessary to confirm that INR or aPTT is within defined limits. However, plasma concentrations of DOACs reached the maximum level at 1 to 4 hours after dosing, and INR or aPTT is often within normal ranges immediately after dosing. Thus, whether or not they are taking DOACs or the last time they took DOACs must be identified in determining patient eligibility for intravenous alteplase. Special care should be taken in determining whether the potential benefits of intravenous alteplase outweigh the potential risks in patients on regular DOAC medication until approximately half a day after the time of the last dose, because DOACs have a half-life of approximately 12 hours. It is likely that eligibility criteria for DOACs will be modified early based on future research outcomes.
\end{abstract}

Modified from Minematsu et al., with permission from Elsevier. ${ }^{13}$

*Conditions requiring careful administration refer to those where treatment may be considered, but where adverse drug reactions are likely to occur and favorable outcomes may not always be achieved. Treatment may be initiated in patients with such conditions only if it is considered by the treating physician that the potential benefits outweigh the possible risks and after they or their legally acceptable representatives provide informed consent.

\section{Follow-up surveys after the second edition}

From our single-center National Cerebral and Cardiovascular Center (NCVC) Stroke Registry, ${ }^{24-26}$ a total of 22 patients (three women, median 75 years old) who visited our emergency room within 4.5 hours after symptom onset and received IVT or acute endovascular therapy, including percutaneous transluminal angioplasty in one patient and mechanical thrombectomy in the others, were studied (unpublished data). Table 2 shows the demographic characteristics, therapeutic process, and outcomes of these patients. Figure 1 shows the relationship between the time from the last dose of DOACs to initiation of IVT (or arterial puncture for patients undergoing direct endovascular therapy: dosing-to-treatment time), levels of aPTT or INR, and clinical outcomes. In our institute, aPTT is measured using the Actin FSL (Siemens Healthcare Diagnostics Inc., Tarrytown, NY, USA); mean aPTT in non-anticoagulated patients is approximately 27 to 28 seconds, and 40 seconds is roughly regarded as 1.5 times the baseline value. Nine patients received direct endovascular therapy by skipping IVT (triangular markers in the figure); three of them showed higher levels of aPTT or INR than the recommended cut-off levels, two skipped IVT due to a recent history of surgery, and the other four skipped IVT mainly because the dosing-to-treatment time was approximately $<6$ hours and close to the expected peak concentration time of DOACs.
ICH occurred in four patients (18\%, markers with red rims). One patient developed a symptomatic subarachnoid hemorrhage, and the other three did not show any neurological deterioration. One patient developed ICH after direct thrombectomy, and the other three developed ICH after IVT plus thrombectomy; the thrombolysis in cerebral infarction scale after thrombectomy was 0 in the patient with symptomatic subarachnoid hemorrhages. The dosing-to-treatment time in the four ICH patients varied between 3.3 and 12.8 hours. When the patients were divided in half depending on the median 6 hours for the dosing-to-treatment time, three patients (27\%) in the shorter-time group versus one (9\%) in the longer-time group had ICH. Nine patients with a modified Rankin Scale (mRS) score of 0 to 2 at 3 months had wide variation of the dosingto-treatment time, between 3.6 and 83.3 hours (median 17.1 hours). On the other hand, four patients with a mRS score of 5 to 6 at 3 months had relatively narrow variation between 3.3 and 6.5 hours (median 4.8 hours).

A multicenter survey involving 118 stroke centers was performed in 2015, including 100 patients (44 women, $76 \pm 10$ years old) who developed ischemic stroke while taking DOACs and received acute reperfusion therapy. ${ }^{27}$ The patients did not overlap with our single-center cases. Of these, 56 patients received IVT alone, 29 received endovascular therapy alone, and the other 15 received both. Twenty patients (20\%) developed ICH within 24 
Table 2. Clinical features of stroke patients taking direct oral anticoagulants who underwent acute reperfusion therapy: the NCVC Stroke Registry

\begin{tabular}{|c|c|c|c|}
\hline & $\begin{array}{l}\text { Intravenous alteplase } \\
\qquad(n=8)\end{array}$ & $\begin{array}{l}\text { Endovascular therapy alone } \\
\qquad(\mathrm{n}=9)\end{array}$ & $\begin{array}{l}\text { Intravenous alteplase and endovas- } \\
\text { cular therapy combined }(n=5)\end{array}$ \\
\hline Women & $0(0)$ & $2(22)$ & $1(20)$ \\
\hline Age (yr) & 73 (56-89) & 76 (48-90) & $75(65-91)$ \\
\hline DOACs & $\begin{array}{c}\text { Dabigatran in } 3, \text { apixaban in } 2, \\
\text { edoxaban in } 3\end{array}$ & $\begin{array}{c}\text { Dabigatran in } 4, \text { rivaroxaban in } 4, \\
\text { edoxaban in } 2\end{array}$ & $\begin{array}{l}\text { Dabigatran in 1, } \\
\text { apixaban in } 4\end{array}$ \\
\hline Indication for DOAC medication & Identified NVAF: 8 & $\begin{array}{c}\text { Identified NVAF: } 7 \\
\text { Suspicion of NVAF: } 1 \text {, DVT: } 1\end{array}$ & $\begin{array}{l}\text { Identified NVAF: } 4 \\
\text { Suspicion of NVAF: } 1\end{array}$ \\
\hline Combined use of antiplatelets & $0(0)$ & $0(0)$ & $1(20)$ \\
\hline Initial aPTT for dabigatran users (sec) & $31(29-31)$ & $34.5(30-44)$ & 33 \\
\hline Initial INR for Xa inhibitor users & $1.12(1.02-1.43)$ & $1.19(0.94-2.91)$ & $1.23(1.16-1.36)$ \\
\hline Anterior territory stroke & 7 (88) & 7 (78) & $5(100)$ \\
\hline DWI-ASPECTS* & $8(5-10)$ & $7(4-10)$ & $7.5(7-10)$ \\
\hline Dosing-to-treatment time (hr) & $15.8(5.6-83.3)$ & $4.5(2.4-26.8)$ & $6.5(3.3-22.9)$ \\
\hline Onset-to-treatment time (hr) & $1.7(1.0-3.6)$ & $2.4(1.5-4.1)$ & $2.1(1.1-3.5)$ \\
\hline $\mathrm{TICl}(0 / 1 / 2 \mathrm{a} / 2 \mathrm{~b} / 3)$ & - & $1 / 1 / 1 / 2 / 4$ & $2 / 0 / 0 / 3 / 0$ \\
\hline \multicolumn{4}{|l|}{$\begin{array}{l}\text { National Institutes of Health Stroke Scale } \\
\text { score }\end{array}$} \\
\hline At baseline & $15.5(1-20)$ & $21(5-31)$ & $16(13-33)$ \\
\hline At $24 \mathrm{hr}$ & $4(0-20)$ & $4(3-22)$ & $15(9-30)$ \\
\hline At 7 day & $2(0-20)$ & $4(0-17)$ & $16(5-31)$ \\
\hline Any ICH $<36$ hr & $0(0)$ & $1(11)$ & $3(60)$ \\
\hline Symptomatic ICH $<36 \mathrm{hr}^{+}$ & $0(0)$ & $0(0)$ & $1(20)^{+}$ \\
\hline $\begin{array}{l}\text { Modified Rankin Scale score at } 3 \text { months } \\
(0-1 / 2 / 3 / 4 / 5 / 6)\end{array}$ & $3 / 2 / 1 / 1 / 1 / 0$ & $3 / 0 / 1 / 5 / 0 / 0$ & $0 / 1 / 1 / 0 / 1 / 2^{\S}$ \\
\hline
\end{tabular}

Values are presented as number (\%) or median (range).

NCVC, National Cerebral and Cardiovascular Center; DOAC, direct oral anticoagulant; NVAF, non-valvular atrial fibrillation; DVT, deep vein thrombosis; aPTT, activated partial thromboplastin time; INR, international normalized ratio; DWI-ASPECTS, diffusion-weighted image Alberta Stroke Program Early CT Score; $\mathrm{TICl}$, thrombolysis in cerebral infarction; $\mathrm{ICH}$, intracranial hemorrhage.

${ }^{*}$ Only for patients with anterior territory stroke; ${ }^{\dagger} \mathrm{CCH}$ with any neurological deterioration; ${ }^{\ddagger}$ Developing subarachnoid hemorrhage; ${ }^{\S}$ One patient died of acute heart failure on day 88 and another of bile duct cancer on day 23.

hours after the therapy; in two patients it was symptomatic, defined as a $\geq 4$-point increase of the National Institutes of Health Stroke Scale (NIHSS) score, and both patients received endovascular therapy alone. The dosing-to-treatment time was available in 52 patients; of these, five of 13 patients (38\%) with a dosingto-treatment time $\leq 4$ hours and four of $39(10 \%)$ with a dosingto-treatment time $>4$ hours had ICH $(P=0.033)$.

Major points derived from the two surveys are: (1) approximately $20 \%$ frequency of post-treatment $\mathrm{ICH}_{\text {; }}(2)$ relatively higher percentage of $\mathrm{ICH}$ in the group with dosing-to-treatment time within 4 to 6 hours; (3) absence of symptomatic ICH after IVT alone; and (4) concentration of patients with death or bedridden state at 3 months in those with dosing-to-treatment time within 6.5 hours, although the poor outcome was not caused by post-treatment ICH in most of them. The last finding may be by chance. The two surveys indicated that IVT for DOAC users according to the second edition of the Japanese guidelines was generally safe, but IVT within 4 to 6 hours after the last dosing might be associated with excess risk of asymptomatic ICH.

\section{A new strategy for acute reperfusion therapy following administration of idarucizumab}

Idarucizumab is a humanized monoclonal antibody fragment that binds dabigatran with high affinity and specificity and rapidly reverses its anticoagulant activity. ${ }^{28}$ On the other hand, idarucizumab does not bind other coagulant factors, does not refill coagulant factors, and has no effects on prothrombin activating ability, platelet aggregation, or endogenous thrombin generation. ${ }^{28,29}$ Thus, it has been thought to have no influence on the coagulation cascade. The agent is in worldwide use based on the results of an interim or final analysis of the Reversal Effects of Idarucizumab on Active Dabigatran (RE-VERSE AD) study. ${ }^{30,31}$ Idaruci- 


\section{O Thrombolysis $\triangle$ Endovascular $\diamond$ Both \\ $\square$ mRS 0-2 $\square$ mRS 3-4 $\square$ mRS 5-6 \\ $\triangle$ Developing intracranial hemorrhage}

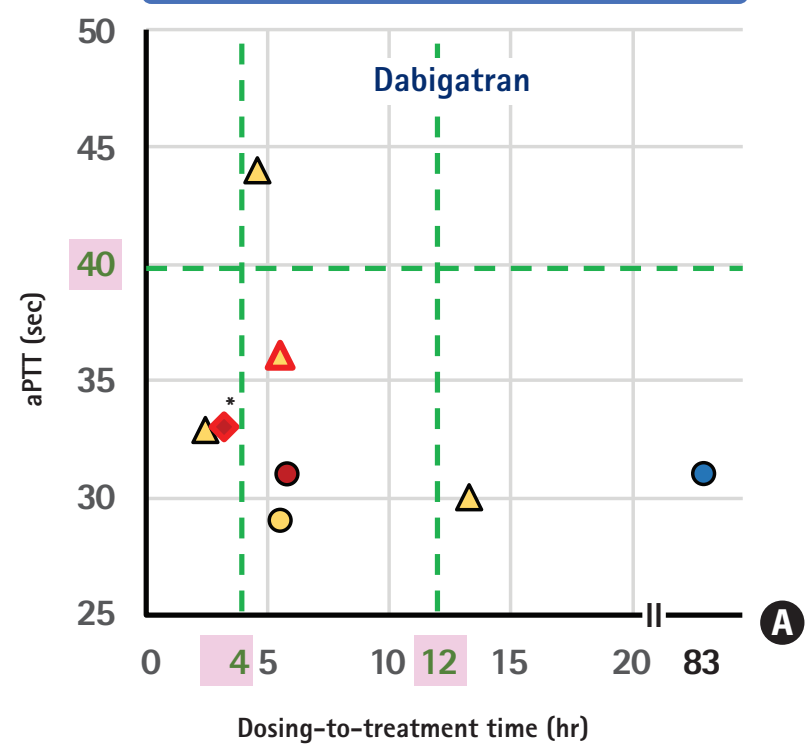

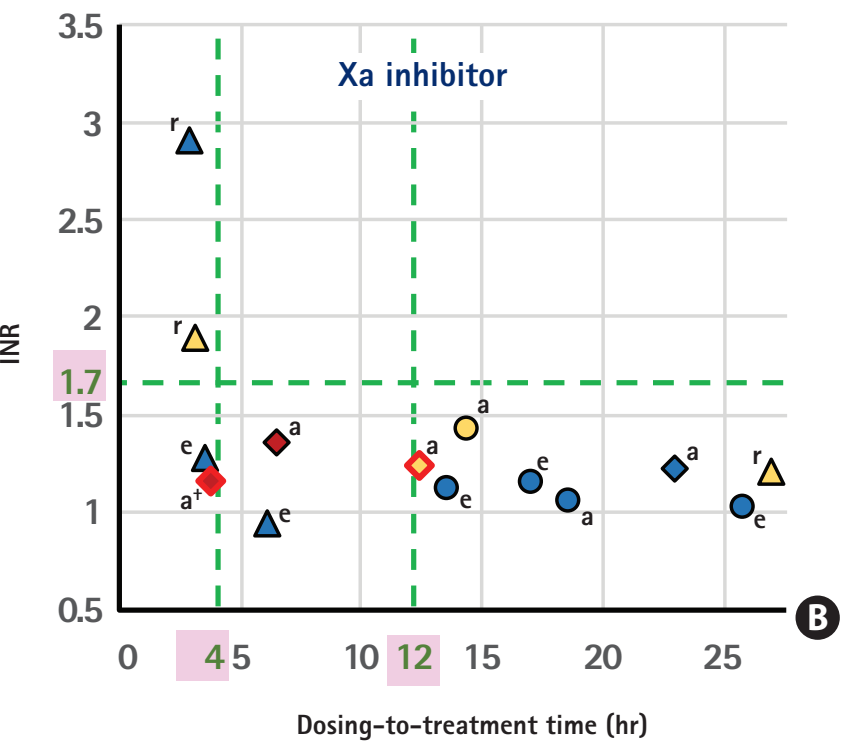

Figure 1. The dosing-to-treatment time, levels of anticoagulant markers, and modified Rankin Scale (mRS) score at 3 months for patients taking dabigatran (A) and Xa inhibitors (B), data from the National Cerebral and Cardiovascular Center (NCVC) Stroke Registry. aPTT, activated partial thromboplastin time; INR, international normalized ratio; a, apixaban; e, edoxaban; r, rivaroxaban. *Seventy-five-year-old man who died of acute heart failure on day 88; ${ }^{\dagger}$ Eighty-fiveyear-old man who developed symptomatic subarachnoid hemorrhage after thrombectomy and died of the bile duct cancer on day 23.

zumab has been in clinical use since November 2016 in Japan.

The specific and rapid effect of idarucizumab as an antidote for dabigatran without an effect on the coagulation cascade may permit us to reverse the activity of dabigatran in patients with hyperacute stroke and then to perform IVT. A total of 47 cases following this new strategy were collected without overlap from 21 articles that were published in English up to February 2018 and could be identified using PubMed with the search terms 'stroke' and 'idarucizumab. ${ }^{132-52}$ One case in our institute was added. Table 3 shows the background characteristics and clinical courses of the 48 cases ( 23 women, median 76.5 years old). Two patients developed ICH, two developed recurrent ischemic stroke, and another one developed deep vein thrombosis and bilateral pulmonary embolism; all of them died or were bedridden. Figure 2 shows the relationships between the baseline NIHSS score, levels of aPT, and outcomes in 42 patients whose data for baseline NIHSS scores were available. Clinical outcomes were assessed mainly at hospital discharge, and independency was identified mainly based on the mRS score (score 0 to 2 was defined as independent) and sometimes estimated based on case description. Interestingly, an aPTT of 40 seconds is likely a good cut-off level to predict outcomes. Two patients with recurrent ischemic events had baseline aPTT levels $<40$ seconds ( 25.9 and
35.5 seconds). The proportion of independent patients was $88 \%$ (14/16) if aPTT $>40$ seconds and 65\% (11/17) if aPTT $<40$ seconds when limited to patients with mild or moderate initial neurological severity with the NIHSS score $<15$ ( $P=0.12$ by the chisquare test); this might suggest the potential enhancement of prothrombic state by idarucizumb in cases with normal or subnormal aPTT levels. We should note that this analysis based on case report series has several limitations including publication bias, retrospective designs, lack of essential data in some cases, and others. Nevertheless, idarucizumab might augment the prothrombotic state to some extent by quick reversal of the anticoagulant effects of dabigatran and might deteriorate neurological deficits, especially when the pre-treatment aPTT is not high enough. The findings raise the question whether the reversal of dabigatran effect prior to IVT is necessary for patients with such a subnormal aPTT.

\section{Revised clinical guides on stroke thrombolysis for anticoagulated patients in Japan}

As compared to numerous case reports from Europe on stroke IVT after administration of idarucizumab, similar reports have 
Table 3. Background characteristics and clinical courses of 48 reported cases receiving idarucizumab immediately prior to intravenous thrombolysis

\begin{tabular}{|c|c|}
\hline Characteristic & \\
\hline Sex category & Women 23, Men 23, NA 2 \\
\hline Age (yr) & Median, 76.5; IQR, 66-84; range, 40-94 \\
\hline Region & Europe 35, East Asia 4, Oceania 8, USA 1 \\
\hline Indication for dabigatran & Atrial fibrillation 33, cardiomyopathy 1, embolic stroke of undetermined sources 1, NA 13 \\
\hline Dosage of dabigatran & $150 \mathrm{mg}$ bid 15, $110 \mathrm{mg}$ bid 29, $150 \mathrm{mg}$ qd 1, NA 3 \\
\hline Admission aPTT (sec) $(n=42)$ & Median, 41.3; IQR, 34-58; range, 24.3-84 \\
\hline Post-idarucizumab aPTT (sec) (n=18) & Median, 29.3; IQR, 28-33; range, 24.1-38.5 \\
\hline Admission NIHSS score $(n=47)$ & Median, 9; IQR, 5-12; range, 3-34 \\
\hline Post-treatment NIHSS score $(n=44)$ & Median, 2; IQR, 0-3; range, 0-42 (death) \\
\hline Combined mechanical thrombectomy & 2: One patient died on day $5 .{ }^{42, *}$ Another recovered to normal. ${ }^{49}$ \\
\hline Post-treatment intracranial hemorrhage & $\begin{array}{l}\text { 2: One patient developed sICH on day } 2 \text { and died on day } 4 .{ }^{38} \text { Another developed sICH with a mRS score of } 5 \text { at } \\
\text { day } 90 .{ }^{49}\end{array}$ \\
\hline Recurrent ischemic stroke in acute stage & 2: Both patients showed early neurological deterioration with discharge mRS scores of $5.33,38,42$ \\
\hline Systemic embolism in acute stage & 1: He developed deep vein thrombosis and bilateral pulmonary embolism and died on day $5{ }^{42, *}$ \\
\hline Clinical outcome mainly at discharge & Independent (mRS score 0-2) 30, dependent (mRS score 3-5) 14, death 3, NA 1 \\
\hline
\end{tabular}

Based on data from references 32-52 and our case record.

IQR, interquartile range; NA, not applicable; bid, twice a day; qd, once a day; aPTT, activated partial thromboplastin time; NIHSS, National Institutes of Health Stroke Scale; sICH, symptomatic intracranial hemorrhage; mRS, modified Rankin Scale.

*The same patient from reference. ${ }^{43}$

\section{O Independent $\Delta$ Dependent $\diamond$ Death \\ $\Delta$ Developing intracranial hemorrhage $\triangle$ Developing ischemic stroke/systemic embolism}

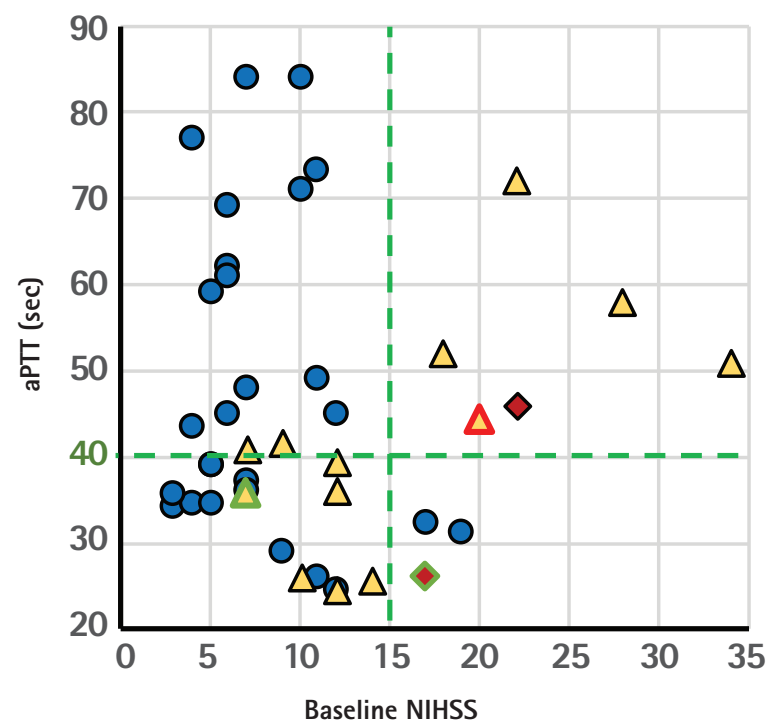

Figure 2. Baseline National Institutes of Health Stroke Scale (NIHSS) score, levels of activated partial thromboplastin time (aPTT), and clinical outcomes of reported cases. Based on data from references 32-52 and our case record. Six patients are missing due to lack of data on aPTT. not been published from Japan up to February 2018. A postmarketing survey of idarucizumab in Japan during the initial 6 months of its clinical use up to May 2017 did not include any patients for emergent use prior to IVT among a total of 130 registered patients. This lack may be due to the relatively wide indication for direct IVT in Japan that patients with aPTT $\leq 40$ seconds could undergo IVT without reversing the anticoagulant effects, or because physicians hesitated to use idarucizumab in such cases in the absence of domestic clinical guides. To overcome the potential limitation, we convened a private working group, prepared a draft for the guide on stroke IVT for anticoagulated patients, and submitted it to the official journal of the Japan Stroke Society. Finally, the society recommended us to re-edit the entire contents of the draft as the authorized clinical guides by the society by adding more experts to the writing group. Table 4 shows the headline recommendations of the guides. ${ }^{14}$

The first major point of change from the previous version is that IVT is not recommended if the time of the last dose of DOAC is $<4$ hours, regardless of the level of aPTT or INR. The revision was partly based on the findings of the multicenter survey indicating that asymptomatic ICH was relatively common in patients with this time window. ${ }^{27}$

The second major point is the complete revision of the recommendation for patients taking dabigatran as follows: 
Table 4. New recommendations on stroke thrombolysis for anticoagulated patients in the new Japanese clinical guides

- For patients taking warfarin

1. Intravenous thrombolysis (IVT, alteplase, $0.6 \mathrm{mg} / \mathrm{kg}$ ) is not recommended if international normalized ratio (INR) of prothrombin time exceeds 1.7 .

2. IVT after emergent reversal of prolonged INR using prothrombin complex concentrates is not recommended. Prothrombin complex concentrates should not be used for patients with hyperacute ischemic stroke, since they can potentially enhance the coagulation cascade and deteriorate patients' neurological deficits.

- For patients during heparinization

3. IVT is not recommended if activated partial thromboplastin time (aPTT) exceeds 1.5 times the baseline value ( $\geq 40$ seconds only as a guide).

4. IVT after emergent reversal of prolonged aPTT using protamine sulfate is not recommended. Protamine should not be used for patients with hyperacute ischemic stroke, since it can potentially enhance the coagulation cascade and deteriorate patients' neurological deficits.

- For patients taking dabigatran

5. Up to now, sensitive markers for the intensity of the dabigatran effect have not been in wide clinical use. IVT is not recommended if aPTT exceeds at least 1.5 times the baseline value ( $\geq 40$ seconds only as a guide).

6. Plasma concentrations of dabigatran reach the maximum level at 1 to 4 hours after dosing, and aPTT is often within normal ranges immediately after dosing. Thus, IVT is not recommended if the time of the last dose is $<4$ hours, regardless of the level of aPTT (IVT can be considered if the time of the last dose is $\geq 4$ hours and the level of aPTT is $\leq 1.5$ times the baseline value).

7. For patients who are regarded as ineligible for IVT based on the above no. 5 or 6 , IVT can be considered after intravenous administration of idarucizumab. However, this recommendation lacks sufficient supporting evidence. Thus, direct mechanical thrombectomy without idarucizumab and without bridging IVT may be reasonable to be considered in institutes capable of performing endovascular stroke treatment.

- For patients taking factor $\mathrm{Xa}$ inhibitors

8. Up to now, sensitive markers for the intensity of Xa inhibitors (rivaroxaban, apixaban, edoxaban) have not been in wide commercial use. IVT is not recommended if INR exceeds at least 1.7.

9. Plasma concentrations of Xa inhibitors reach the maximum level at 1 to 4 hours after dosing, and INR is often within normal ranges immediately after dosing. Thus, IVT is not recommended if the time of the last dose is $<4$ hours, regardless of the level of INR (direct mechanical thrombectomy can be considered for such patients in institutes capable of performing endovascular stroke treatment; IVT can be considered if the time of the last dose is $\geq 4$ hours and the level of INR is $\leq 1.7$ ).

10. IVT after emergent reversal of prolonged INR using antidotes for other anticoagulants is not recommended.

- Careful determination of eligibility for reperfusion therapy in overall anticoagulated patients

11. Eligibility for IVT should be determined with care for patients taking anticoagulants regardless of their intensity. It should be considered if potential benefits outweigh the possible risks, especially when the time of the last dose of dabigatran or Xa inhibitor is $<12$ hours, because these anticoagulants have a half-life of approximately 12 hours.

12. Eligibility for mechanical thrombectomy should be determined with care if potential benefits outweigh the possible risks. Mechanical thrombectomy should be performed according to the official package insert for each device.

Adapted from Toyoda et al., with permission from Japan Stroke Society. ${ }^{14}$

Revised from reference, ${ }^{14}$ with additional description between parentheses.

(1) IVT can be considered without pretreatment with idarucizumab if aPT is $\leq 40$ seconds and the time of the last dose is $>4$ hours. Additional mechanical thrombectomy can be considered if indicated.

(2) Direct mechanical thrombectomy can be considered without pretreatment with idarucizumab or rt-PA if eligible, when aPTT is $>40$ seconds or the time of the last dose is or may be $\leq 4$ hours.

(3) Treatment with $5 \mathrm{~g}$ idarucizumab, followed immediately by IVT, can be considered if mechanical thrombectomy cannot be quickly performed for patients whose aPTT is $>40$ seconds or the time of the last dose is or may be $\leq 4$ hours. A blood sample should be collected and sent to the laboratory immediately after initiating IVT. If aPTT is not normalized, IVT should be terminated immediately.

The strategy is also shown as a flowchart in Figure 3. We also recommend that the eligibility for idarucizumab usage can be determined based on findings of markers sensitive to the intensity of dabigatran, such as ecarin clotting time, in institutes where emergent assessment of these markers is available.

Since aPTT does not completely reflect the intensity of dabigatran, treatment with idarucizumab prior to IVT when aPTT $<40$ seconds would further decrease the risk of post-IVT ICH. However, we have concerns that emergent reversal of dabigatran's effects might enhance prothrombotic states to some extent and deteriorate neurological severity, as discussed earlier. This is the major reason for us not to require idarucizumab in patients with aPTT $<40$ seconds.

It is instructive to compare our revised guides with an expert opinion from European researchers. ${ }^{53}$ The European expert opinion targeted patients meeting the following criteria: (1) pretreatment with dabigatran; (2) last intake of dabigatran $<24$ hours (96 hours if creatinine clearance $<30 \mathrm{~mL} / \mathrm{min}$ ); (3) acute ischemic stroke (bleeding excluded by computed tomography or magnetic resonance imaging) $<4.5$ hours; and (4) no additional contraindications for rt-PA. Direct mechanical thrombectomy is preferentially considered for such patients, and when they cannot receive thrombectomy, treatment with idarucizumab, fol- 
$<4.5$ hours after onset?

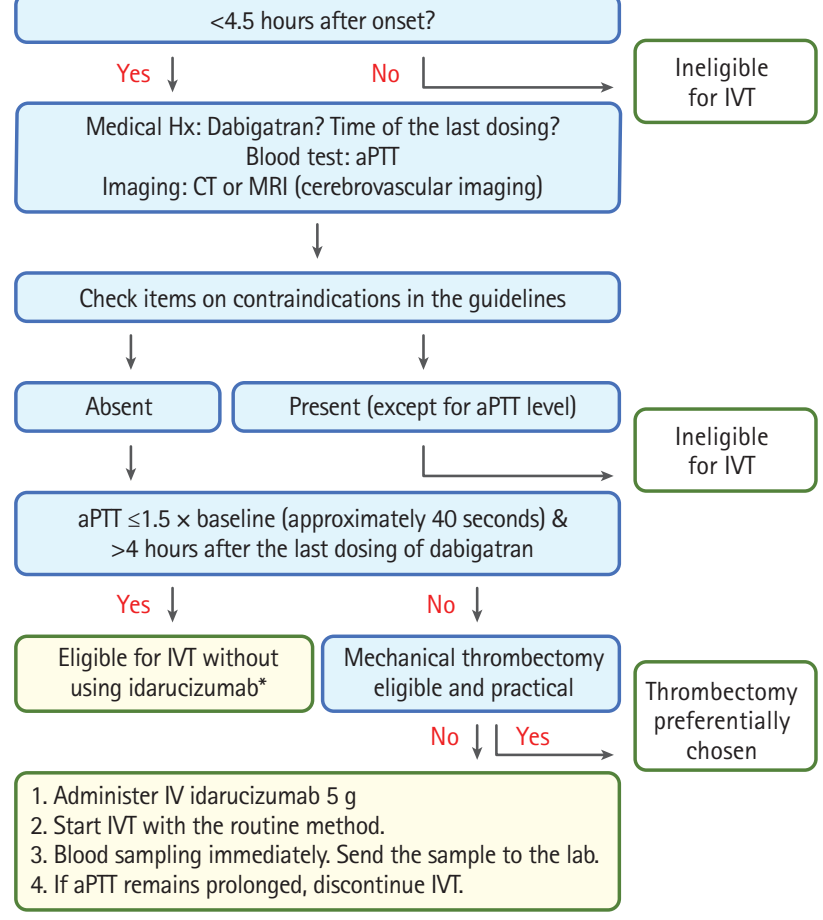

Figure 3. A flowchart on the strategy of stroke thrombolysis for patients taking dabigatran in the new Japanese clinical guides. Direct mechanical thrombectomy can be considered for some of patients ineligible for intravenous thrombolysis. Adapted from Toyoda et al., with permission from Japan Stroke Society. ${ }^{14} \mathrm{IVT}$, intravenous thrombolysis; $\mathrm{Hx}$, history; aPTT, activated partial thromboplastin time; CT, computed tomography; MRI, magnetic resonance imaging.

lowed immediately by IVT, can be considered regardless of the aPTT level. In another expert opinion from the French Study Group on Hemostasis and Thrombosis, treatment with idarucizumab prior to IVT is recommended when the concentration of dabigatran is $>100 \mathrm{ng} / \mathrm{mL}$, idarucizumab is not necessary when the concentration is $<50 \mathrm{ng} / \mathrm{mL}$, and use of idarucizumab should be discussed in terms of the individual risk/benefit ratio and the possibility of mechanical thrombectomy when the concentration of dabigatran is between 50 and $100 \mathrm{ng} / \mathrm{mL}^{54}$

In addition to recommendations for IVT after idarucizumab, we briefly refer to recommendations for IVT after other antidotes in our new guides. ${ }^{14}$ For example, four-factor prothrombin complex concentrates refill coagulation factors II, VII, IX, and $\mathrm{X}$ and can prevent hematoma expansion in patients with $\mathrm{ICH}$ related to vitamin $\mathrm{K}$ antagonists. ${ }^{55,56}$ In contrast to the role of idarucizumab for dabigatran, the effect of prothrombin complex concentrates as antidotes is non-specific, and the concentrates accordingly augment the coagulation cascade and may deteriorate neurological deficits. Thus, we do not recommend IVT after emergent reversal of prolonged INR using the concentrates at this time, although a few case reports sup- ported the feasibility of this strategy. ${ }^{57,58}$ Similarly, we do not recommend IVT after emergent reversal of prolonged aPTT using protamine sulfate, though a few case reports supported its feasibility. ${ }^{59,60}$

In the present review, we do not fully introduce recommendations of mechanical thrombectomy in Japan. We recently revised Japanese guidelines for application of devices on mechanical thrombectomy (http://www.jsts.gr.jp/img/noukessen_3.pdf [in Japanese]); the English version will be published soon.

\section{Conclusions}

In this review, the details and rationale of the new clinical guides on stroke IVT for anticoagulated patients in Japan are introduced. The most unique point of the guides is the use of traditional coagulation markers such as aPTT as cut-offs for idarucizumab use before IVT. These guides are practical and to some extent economical, but they have some limitations, including lack of corroborating information from sufficient numbers of relevant cases, especially from Japan. It is expected that there will be more stroke patients treated with idarucizumab prior to IVT according to the guides. The recommendations in the present guides will be further modified based on the results of future research for the third editions of the Japanese guidelines on stroke thrombolysis that will be hopefully published in 2019.

\section{Disclosure}

Kazunori Toyoda has received honoraria (moderate, $<10 \mathrm{KUSD} /$ y) from Bayer, BMS, Boehringer Ingelheim, Daiichi Sankyo, and Takeda Yakuhin. Hiroshi Yamagami has received honoraria (moderate) from Bayer and Daiichi Sankyo and research support (moderate) from BMS.

\section{Acknowledgments}

The authors would like to thank Drs. Yasuyuki Iguchi (Jikei University School of Medicine, Tokyo), Shigeru Nogawa (Tokai University Hachioji Hospital, Hachioji), Toshiyuki Miyata (National Cerebral and Cardiovascular Center [NCVC], Suita), and Yasuhiro Hasegawa (St Marianna University School of Medicine, Kawasaki) for their contributions with the authors as writing committee members of the Japanese guides on stroke thrombolysis for anticoagulated patients. The authors would also like to thank Drs. Masafumi Ihara, Masayuki Shiozawa, and Takeshi Yoshimoto (NCVC, Suita) for providing data on the NCVC Stroke Registry.

This review article was supported, in part, by Intramural Re- 
search Funds (H28-4-1, H29-3-3) for Cardiovascular Diseases of the NCVC, Grants from the Japan Agency for Medical Research and Development (AMED: 18ek0210091h0002, 18ek0210055h0003), and JSPS KAKENHI JP17H04308.

\section{References}

1. Toyoda K. Pharmacotherapy for the secondary prevention of stroke. Drugs 2009;69:633-647.

2. Toyoda K, Koga M, Hayakawa M, Yamagami H. Acute reperfusion therapy and stroke care in Asia after successful endovascular trials. Stroke 2015;46:1474-1481.

3. Yamaguchi T, Mori E, Minematsu K, Nakagawara J, Hashi K, Saito I, et al. Alteplase at $0.6 \mathrm{mg} / \mathrm{kg}$ for acute ischemic stroke within 3 hours of onset: Japan Alteplase Clinical Trial (JACT). Stroke 2006;37:1810-1815.

4. Shinohara Y, Yamaguchi T. Outline of the Japanese Guidelines for the Management of Stroke 2004 and subsequent revision. Int J Stroke 2008;3:55-62.

5. Yasaka M, Minematsu K, Yamaguchi T. Optimal intensity of international normalized ratio in warfarin therapy for secondary prevention of stroke in patients with non-valvular atrial fibrillation. Intern Med 2001;40:1183-1188.

6. Inoue $\mathrm{H}_{1}$ Okumura $\mathrm{K}$, Atarashi $\mathrm{H}$, Yamashita T, Origasa $\mathrm{H}, \mathrm{Ku}$ magai $\mathrm{N}$, et al. Target international normalized ratio values for preventing thromboembolic and hemorrhagic events in Japanese patients with non-valvular atrial fibrillation: results of the J-RHYTHM Registry. Circ J 2013;77:2264-2270.

7. Hori M, Matsumoto M, Tanahashi N, Momomura S, Uchiyama $\mathrm{S}$, Goto $\mathrm{S}$, et al. Rivaroxaban vs. warfarin in Japanese patients with atrial fibrillation: the J-ROCKET AF study. Circ $J$ 2012;76:2104-2111.

8. Isshiki T, Kimura T, Ogawa H, Yokoi H, Nanto S, Takayama M, et al. Prasugrel, a third-generation $\mathrm{P} 2 \mathrm{Y} 12$ receptor antagonist, in patients with coronary artery disease undergoing elective percutaneous coronary intervention. Circ J2014;78:2926-2934.

9. Takahashi H, Wilkinson GR, Caraco Y, Muszkat M, Kim RB, Kashima $T$, et al. Population differences in S-warfarin metabolism between CYP2C9 genotype-matched Caucasian and Japanese patients. Clin Pharmacol Ther 2003;73:253-263.

10. Rieder MJ, Reiner AP, Gage BF, Nickerson DA, Eby CS, McLeod $\mathrm{HL}$, et al. Effect of VKORC1 haplotypes on transcriptional regulation and warfarin dose. N Engl J Med 2005;352:2285-2293.

11. Shen AY, Chen W, Yao JF, Brar SS, Wang X, Go AS. Effect of race/ethnicity on the efficacy of warfarin: potential implications for prevention of stroke in patients with atrial fibrillation. CNS Drugs 2008;22:815-825.

12. Guideline Committee of the Japan Stroke Society for intra- venous rt-PA (alteplase) in acute ischemic stroke. Guidelines for intravenous application of rt-PA (alteplase), October 2005. Jpn J Stroke 2005;27:327-354.

13. Minematsu K, Toyoda K, Hirano T, Kimura K, Kondo R, Mori E, et al. Guidelines for the intravenous application of recombinant tissue-type plasminogen activator (alteplase), the second edition, October 2012: a guideline from the Japan Stroke Society. J Stroke Cerebrovasc Dis 2013;22:571-600.

14. Toyoda K, Iguchi Y, Koga M, Nogawa S, Hasegawa Y, Miyata $\mathrm{S}$, et al. Updated recommendations on acute reperfusion therapy for anticoagulated patients with ischemic stroke. Jpn J Stroke 2018;40:123-135.

15. Yamaguchi $T$, Kikuchi $H$, Hayakawa $T$; the Japanese Thrombolysis Study Group. Clinical efficacy and safety of intravenous tissue plasminogen activator in acute embolic stroke: a randomized, double-blind, dose-comparison study of duteplase. In: Yamaguchi T, Mori E, Minematsu K, del Zoppo G. Thrombolytic Therapy in Acute Ischemic Stroke III. Tokyo, JP: Springer-Verlag, 1995;223-229.

16. Ueshima $S$, Matsuo 0 . The differences in thrombolytic effects of administrated recombinant t-PA between Japanese and Caucasians. Thromb Haemost 2002;87:544-546.

17. Toyoda K, Koga M, Naganuma M, Shiokawa Y, Nakagawara J,

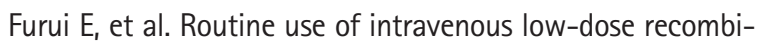
nant tissue plasminogen activator in Japanese patients: general outcomes and prognostic factors from the SAMURAI register. Stroke 2009;40:3591-3595.

18. Mori E, Minematsu K, Nakagawara J, Yamaguchi T, Sasaki M, Hirano $\mathrm{T}$, et al. Effects of $0.6 \mathrm{mg} / \mathrm{kg}$ intravenous alteplase on vascular and clinical outcomes in middle cerebral artery occlusion: Japan Alteplase Clinical Trial II (J-ACT II). Stroke 2010;41: 461-465.

19. Nakagawara J, Minematsu K, Okada Y, Tanahashi N, Nagahi-

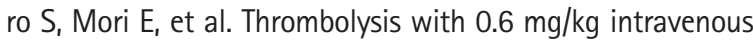
alteplase for acute ischemic stroke in routine clinical practice: the Japan post-Marketing Alteplase Registration Study (J-MARS). Stroke 2010;41:1984-1989.

20. Anderson CS, Robinson T, Lindley RI, Arima H, Lavados PM, Lee $\mathrm{TH}$, et al. Low-dose versus standard-dose intravenous alteplase in acute ischemic stroke. N Eng/ J Med 2016;374: 2313-2323.

21. Xian Y, Liang L, Smith EE, Schwamm LH, Reeves MJ, Olson DM, et al. Risks of intracranial hemorrhage among patients with acute ischemic stroke receiving warfarin and treated with intravenous tissue plasminogen activator. JAMA 2012;307: 2600-2608.

22. Steiner $T$, Böhm $M$, Dichgans $M$, Diener HC, Ell C, Endres $M$, et al. Recommendations for the emergency management of complications associated with the new direct oral anticoag- 
ulants (DOACs), apixaban, dabigatran and rivaroxaban. Clin Res Cardiol 2013;102:399-412.

23. Diener HC, Foerch C, Riess H, Röther J, Schroth G, Weber R. Treatment of acute ischaemic stroke with thrombolysis or thrombectomy in patients receiving anti-thrombotic treatment. Lancet Neurol 2013;12:677-688.

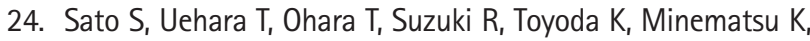
et al. Factors associated with unfavorable outcome in minor ischemic stroke. Neurology 2014;83:174-181.

25. Hashimoto T, Hayakawa M, Funatsu N, Yamagami H, Satow T, Takahashi JC, et al. Histopathologic analysis of retrieved thrombi associated with successful reperfusion after acute stroke thrombectomy. Stroke 2016;47:3035-3037.

26. Toyoda $K$, Koga M, Yamagami H, Yokota C, Sato S, Inoue $M$, et al. Seasonal variations in neurological severity and outcomes of ischemic stroke: 5 -year single-center observational study. Circ J 2018;82:1443-1450.

27. Suzuki K, Aoki J, Sakamoto Y, Abe A, Suda S, Okubo S, et al. Low risk of ICH after reperfusion therapy in acute stroke patients treated with direct oral anti-coagulant. J Neurol Sci 2017;379:207-211.

28. Schiele F, van Ryn J, Canada K, Newsome C, Sepulveda E, Park J, et al. A specific antidote for dabigatran: functional and structural characterization. Blood 2013;121:3554-3562.

29. Schmohl M, Glund S, Harada A, Imazu S, De Smet M, Mos-

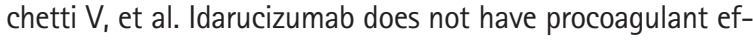
fects: assessment of thrombosis biomarkers in healthy volunteers. Thromb Haemost 2017;117:269-276.

30. Pollack CV Jr, Reilly PA, Eikelboom J, Glund S, Verhamme P, Bernstein RA, et al. Idarucizumab for dabigatran reversal. $N$ Engl J Med 2015;373:511-520.

31. Pollack CV Jr, Reilly PA, van Ryn J, Eikelboom JW, Glund S, Bernstein RA, et al. Idarucizumab for dabigatran reversal: full cohort analysis. N Engl J Med 2017;377:431-441.

32. Schäfer N, Müller A, Wüllner U. Systemic thrombolysis for ischemic stroke after antagonizing dabigatran with idarucizumab: a case report. J Stroke Cerebrovasc Dis 2016;25:e126e127.

33. Berrouschot J, Stoll A, Hogh T, Eschenfelder CC. Intravenous thrombolysis with recombinant tissue-type plasminogen activator in a stroke patient receiving dabigatran anticoagulant after antagonization with idarucizumab. Stroke 2016;47:1936-1938.

34. Kafke W, Kraft P. Intravenous thrombolysis after reversal of dabigatran by idarucizumab: a case report. Case Rep Neurol 2016;8:140-144.

35. Gawehn A, Ayari Y, Heuschkel C, Kaste M, Kermer P. Successful thrombolysis with recombinant tissue plasminogen activator after antagonizing dabigatran by idarucizumab: a case report. J Med Case Rep 2016;10:269.

36. Schulz JG, Kreps B. Idarucizumab elimination of dabigatran minutes before systemic thrombolysis in acute ischemic stroke. J Neurol Sci 2016;370:44.

37. Mutzenbach JS, Pikija S, Otto F, Halwachs U, Weymayr F, Sellner J. Intravenous thrombolysis in acute ischemic stroke after dabigatran reversal with idarucizumab: a case report. Ann Clin Transl Neurol 2016;3:889-892.

38. Turine G, Peeters A, Hermans C, Eeckhoudt S, Duprez T. Intravenous thrombolysis after reversal of dabigatran by idarucizumab: a moment to be a pioneer. Acta Neurol Belg 2017; 117:753-755.

39. Ng FC, Bice J, Rodda A, Lee-Archer M, Crompton DE. Adverse clinical outcomes after dabigatran reversal with idarucizumab to facilitate acute stroke thrombolysis. J Neurol 2017; 264:591-594.

40. Facchinetti R, DeGuidi G, Pitoni F, Ricci G, Lippi G. Rapid and well tolerated action of idarucizumab for antagonizing dabigatran in a patient needing urgent thrombolysis: a case report. Blood Coagul Fibrinolysis 2017;28:576-579.

41. Tireli D, He J, Nordling MM, Wienecke T. Systemic thrombolysis in acute ischemic stroke after dabigatran etexilate reversal with idarucizumab: a case report. J Stroke Cerebrovasc Dis 2017;26:e123-e125.

42. Cappellari M, Forlivesi S, Squintani GM, Facchinetti R, Bovi P. Intravenous thrombolysis for stroke after dabigatran reversal with idarucizumab: an update. J Thromb Thrombolysis 2017; 43:528-529.

43. Kermer $\mathrm{P}$, Eschenfelder CC, Diener HC, Grond $\mathrm{M}$, Abdalla $\mathrm{Y}, \mathrm{Al}-$ thaus $K_{1}$ et al. Antagonizing dabigatran by idarucizumab in cases of ischemic stroke or intracranial hemorrhage in Germany: a national case collection. Int J Stroke 2017;12:383-391.

44. Bissig D, Manjunath R, Traylor BR, Richman DP, Ng KL. Acute stroke despite dabigatran anticoagulation treated with idarucizumab and intravenous tissue plasminogen activator. $J$ Stroke Cerebrovasc Dis 2017;26:e102-e104.

45. Vosko MR, Bocksrucker C, Drwiła R, Dulíček P, Hauer T, Mutzenbach J, et al. Real-life experience with the specific reversal agent idarucizumab for the management of emergency situations in dabigatran-treated patients: a series of 11 cases. J Thromb Thrombolysis 2017;43:306-317.

46. Alvarez Bravo G, Orts Castro E, Carvalho Monteiro G, López Zuazo I. Intravenous fibrinolysis in ischemic stroke of large vessel after reversing effect of dabigatran with idarucizumab. J Stroke Cerebrovasc Dis 2017;26:e192-e193.

47. Agosti S, Casalino L, Rocci E, Zaccone G, Rota E. Successful intravenous thrombolysis for ischemic stroke after reversal of dabigatran anticoagulation with idarucizumab: a case report. 
J Med Case Rep 2017;11:224.

48. von Wowern FA, Brizzi M, Holst J. Idarucizumab in three patients needing urgent surgical intervention and one case of intravenous thrombolysis in ischaemic stroke. Eur J Case Rep Intern Med 2017;4:1-3. https://doi.org/10.12890/2017_000569.

49. Pikija S, Sztriha LK, Sebastian Mutzenbach J, Golaszewski SM, Sellner J. Idarucizumab in dabigatran-treated patients with acute ischemic stroke receiving alteplase: a systematic review of the available evidence. CNS Drugs 2017;31:747-757.

50. Tse DM, Young L, Ranta A, Barber PA. Intravenous alteplase and endovascular clot retrieval following reversal of dabigatran with idarucizumab. J Neurol Neurosurg Psychiatry2018;89:549-550.

51. Tsai LK, Lin HJ, Chua SK, Liao PC, Yang YP, Chou PC, et al. Real-world experience with idarucizumab to reverse anticoagulant effect in dabigatran-treated patients: report of 11 cases from Taiwan. J Stroke Cerebrovasc Dis 2018;27:e27-e33.

52. Lo WT, Ng KF, Chan SC, Kwok VW, Fong CS, Chan ST, et al. Intravenous stroke thrombolysis after reversal of dabigatran effect by idarucizumab: first reported case in Hong Kong. Hong Kong Med J 2018;24:81-83.

53. Diener HC, Bernstein R, Butcher K, Campbell B, Cloud G, Davalos $A$, et al. Thrombolysis and thrombectomy in patients treated with dabigatran with acute ischemic stroke: expert opinion. Int J Stroke 2017;12:9-12.

54. Touzé E, Gruel Y, Gouin-Thibault I, De Maistre E, Susen S, Sie P, et al. Intravenous thrombolysis for acute ischaemic stroke in patients on direct oral anticoagulants. Eur J Neurol 2018;25: 747-e52.

55. Steiner T, Poli S, Griebe M, Hüsing J, Hajda J, Freiberger A, et al. Fresh frozen plasma versus prothrombin complex concentrate in patients with intracranial haemorrhage related to vitamin $\mathrm{K}$ antagonists (INCH): a randomised trial. Lancet Neurol 2016;15:566-573.

56. Yasaka M, Brainsky A, Toyoda K. Prothrombin complex concentrate for vitamin $\mathrm{K}$ antagonist-associated intracranial hemorrhage: global evidence and the Japanese perspective. Circ J 2017;81:1564-1573.

57. Jalini S, Jin AY, Taylor SW. Reversal of warfarin anticoagulation with prothrombin complex concentrate before thrombolysis for acute stroke. Cerebrovasc Dis 2012;33:597.

58. Chausson N, Aghasaryan M, Soumah D, Altarcha T, Smadja D. Reversal of vitamin $\mathrm{K}$ antagonist therapy by prothrombin complex concentrate before thrombolysis for acute stroke. Cerebrovasc Dis Extra 2013;3:95-96.

59. Fontaine GV, Smith SM. Alteplase for acute ischemic stroke after heparin reversal with protamine: a case report and review. Pharmacotherapy 2017;37:e103-e106.

60. Danoun O, Sachar P, Rajamani K. Thrombolysis for acute ischemic stroke after protamine reversal of heparin. Am J Ther 2018; 25:e552-e554. 